University of Wollongong

Research Online

Australian Institute for Innovative Materials -

Papers

Australian Institute for Innovative Materials

$1-1-2020$

Novel integrated strategies toward efficient and stable unassisted photoelectrochemical water splitting

Liqun Wang

Wenping Si

Xinggang Hou

Mingxia Wang

Xiaoli Liu

See next page for additional authors

Follow this and additional works at: https://ro.uow.edu.au/aiimpapers

Part of the Engineering Commons, and the Physical Sciences and Mathematics Commons

Research Online is the open access institutional repository for the University of Wollongong. For further information contact the UOW Library: research-pubs@uow.edu.au 


\title{
Novel integrated strategies toward efficient and stable unassisted photoelectrochemical water splitting
}

\author{
Abstract \\ (C) 2020 Unassisted photoelectrochemical (PEC) water splitting for hydrogen evolution has been regarded \\ as a sustainable route for the harvest and utilization of solar energy. To achieve such unassisted PEC \\ water splitting, two novel integrated strategies have been developed: to design tandem structures of \\ photoanodes/photocathodes and to construct hybrid devices of solar/PEC cells. Some key unsolved \\ problems, however, still limit the further development of high-performance unassisted PEC water splitting, \\ such as low efficiency, poor stability, and high cost. Herein, we present a brief summary of the latest \\ development in this area and propose perspectives for further enhancing this state-of-the-art solar- \\ to-hydrogen conversion technology, including all-metal oxide photoelectrodes, nanoarray design, surface \\ modification, device coupling, monolithic configuration, and multi-integration. \\ Disciplines \\ Engineering | Physical Sciences and Mathematics

\section{Publication Details} \\ Wang, L., Si, W., Hou, X., Wang, M., Liu, X., Ye, Y., Hou, F. \& Liang, J. (2020). Novel integrated strategies \\ toward efficient and stable unassisted photoelectrochemical water splitting. Sustainable Materials and \\ Technologies, 25 e00209.

\section{Authors} \\ Liqun Wang, Wenping Si, Xinggang Hou, Mingxia Wang, Xiaoli Liu, Yuhang Ye, Feng Hou, and Ji Liang
}




\title{
Novel integrated strategies toward efficient and stable unassisted photoelectrochemical water splitting
}

\author{
Liqun Wang a,1,*, Wenping Si ${ }^{\text {b,1 }}$, Xinggang Hou ${ }^{\text {a }}$, Mingxia Wang ${ }^{\text {a }}$, Xiaoli Liu ${ }^{a}$, Yuhang Ye ${ }^{\text {a }}$, \\ Feng Hou ${ }^{\mathrm{b}, *}$, Ji Liang $\mathrm{b}^{\mathrm{b}, \mathrm{c}, *}$ \\ a Applied Physics Department, College of Physics and Materials Science, Tianjin Normal University, Tianjin 300387, China \\ ${ }^{\mathrm{b}}$ Key Laboratory of Advanced Ceramics and Machining Technology, Ministry of Education, School of Materials Science and Engineering, Tianjin University, Tianjin 300350, China

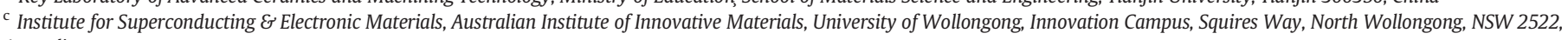 \\ Australia
}

\section{A R T I C L E I N F O}

\section{Article history:}

Received 17 July 2020

Received in revised form 3 August 2020

Accepted 4 August 2020

\section{Keywords:}

Photoelectrochemistry

Water splitting

Hydrogen evolution

\begin{abstract}
A B S T R A C T
Unassisted photoelectrochemical (PEC) water splitting for hydrogen evolution has been regarded as a sustainable route for the harvest and utilization of solar energy. To achieve such unassisted PEC water splitting, two novel integrated strategies have been developed: to design tandem structures of photoanodes/photocathodes and to construct hybrid devices of solar/PEC cells. Some key unsolved problems, however, still limit the further development of high-performance unassisted PEC water splitting, such as low efficiency, poor stability, and high cost. Herein, we present a brief summary of the latest development in this area and propose perspectives for further enhancing this state-of-the-art solar-to-hydrogen conversion technology, including all-metal oxide photoelectrodes, nanoarray design, surface modification, device coupling, monolithic configuration, and multiintegration.
\end{abstract}

() 2020 Published by Elsevier B.V.

\section{Contents}

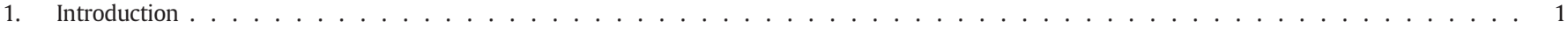

2. Tandem structure design of photoanodes and photocathodes . . . . . . . . . . . . . . . . . . . . . . . . .

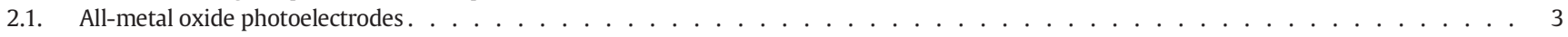

2.2. Nanoarray design . . . . . . . . . . . . . . . . . . . . . . . . . . . . . . . . . . 4

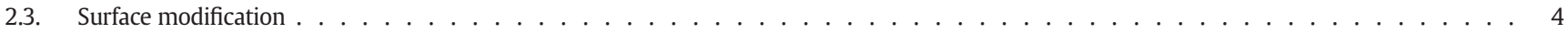

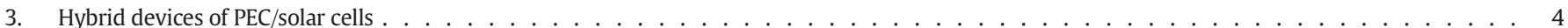

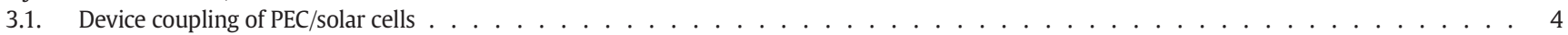

3.2. Monolithic configuration . . . . . . . . . . . . . . . . . . . . . . . . . . . . 4

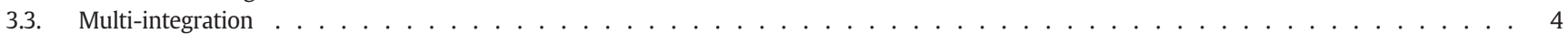

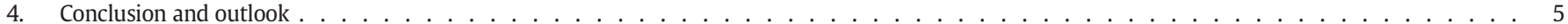

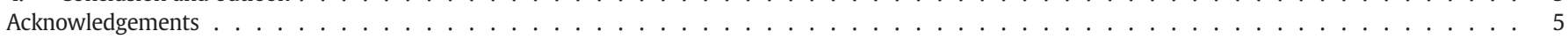

References $\ldots \ldots \ldots$

\footnotetext{
* Corresponding authors.

E-mail addresses: wlxywlq@mail.tjnu.edu.cn (L. Wang), houf@tju.edu.cn (F. Hou), liangj@uow.edu.au (J. Liang).

${ }^{1}$ These authors contribute equally to this paper.
}

\section{Introduction}

Since the first industrial revolution in the mid-eighteenth century, traditional fossil fuels (e.g., coal, oil, and natural gas) have greatly promoted the development of the societies' productivity. With the rapid industrialization and urbanization in recent decades, nevertheless, fossil fuels have been excessively exploited and consumed. It inevitably 
leads to two major crises, including the energy shortage and environmental deterioration [1-3]. This is because fossil fuels have a limited reserve on the earth and will soon be exhausted according to their current annual consumption rates. Besides, the combustion of fossil fuels also releases a huge amount of toxic/green-house gases and harmful particle matters, resulting in severe environmental issues, such as global warming, acid rain, ozone depletion, smog, and so on [4,5].

In order to tackle these problems, a sustainable strategy is to capture and utilize renewable and clean energy sources (i.e., new energy sources), including solar energy [6-8], wind energy [9], nuclear energy [10], geothermal energy [11], wave/tidal energy [12,13], biomass energy [14], natural gas hydrates [15], etc., to replace the aforementioned fossil fuels. Among the various types of new energy sources, solar energy has been regarded as a most promising alternative of the carbon-based fossil fuels because of its unique merits, summarized as following [16-18]:

i) Abundance. The energy carried by sunlight reaching the earth's surface is $\sim 5.4 \times 10^{21} \mathrm{kWh}$ per year, which is about 5000 times of the global annual energy consumption. That is to say, even if $0.02 \%$ of solar energy is effectively used, human energy demand can be satisfied.

ii) Sustainability. Based on the rate of hydrogen fusion in the Sun, its hydrogen reserves can be consumed continuously for more than $6.0 \times 10^{10}$ years, meaning that solar energy is an inexhaustible and renewable energy resource for us.

iii) Clean energy. Unlike traditional fossil fuels, solar energy virtually does not produce any pollution. Thus, solar energy can be considered as green energy, which is very beneficial for protecting the ecological environment.

iv) Convenience. In theory, solar energy can be harvested and further utilized wherever the sunlight can shine, which is especially facile for the remote areas. Besides, miniaturized and flexible solar cells also provide a great possibility for the development of self-powered portable devices.

Although solar energy exhibits the above-mentioned advantages, its inherent shortcomings, including intermittence, instability, and low power density, still impede the large-scale commercial application $[19,20]$. To achieve the efficient utilization of solar energy, the conversion of solar energy into hydrogen fuel (i.e., hydrogen gas) has become a potential solution in this field [21]. Specifically, as an ideal carrier for solar energy, hydrogen fuel shows a serial of excellent properties, such as carbon-free and none-emission features, high energy density $\left(\sim 140 \mathrm{MJ} \mathrm{kg}{ }^{-1}\right)$, storability (e.g., hydrogen storage alloys and liquid hydrogen), and mature technology for electricity generation (e.g., hydrogen fuel cells and hydrogen gas steam boilers) [22-26]. Consequently, the solar-to-hydrogen ( $\mathrm{STH}$ ) conversion can not only overcome the drawbacks of solar energy but also provide a feasible way for the large-scale and long-term development of solar energy.

As one typical artificial photosynthesis process, photoelectrochemical (PEC) water splitting has been considered as a highly promising strategy for the STH conversion, in terms of its relatively high energy conversion efficiency, environmentally-friendly process, and abundant hydrogen resource [27-33]. A typical PEC water splitting process can be achieved in a PEC cell, commonly with two solar-driven half-reactions: hydrogen evolution reaction (HER) and oxygen evolution reaction (OER), which are carried out at surfaces of (photo)anodes and (photo)cathodes, respectively (Eqs. (1) and (2)).

HER at (photo) cathode : $2 \mathrm{H}^{+}+2 \mathrm{e}^{-} \rightarrow \mathrm{H}_{2} E_{\text {red }}{ }^{0}=0 \mathrm{~V}$

OER at (photo)anode : $2 \mathrm{H}_{2} \mathrm{O}+4 \mathrm{~h}^{*} \rightarrow 4 \mathrm{H}^{+}+\mathrm{O}_{2} \mathrm{E}_{\mathrm{oX}}{ }^{0}=1.23 \mathrm{~V}$

where, $E_{\mathrm{red}}^{0}$ and $E_{\mathrm{ox}}^{0}$ are $\mathrm{H}_{2}$ evolution potential $(0 \mathrm{~V} v$ s. normal hydrogen electrode $(\mathrm{NHE})$ at $\mathrm{pH}=0$ ) and $\mathrm{O}_{2}$ evolution potential ( $1.23 \mathrm{~V} v \mathrm{~s}$. NHE at $\mathrm{pH}=0)$, respectively.
Theoretically, as long as the bandgap of photoelectrode is equal to or larger than the minimum energy for decomposing water molecules (1.23 eV), photogenerated electrons and holes produced on photoelectrodes under illumination can drive the HER and OER, respectively. Unfortunately, undesirable energy loss often exists during the PEC water splitting process, such as over potentials and contact resistances. As a result, an external bias is commonly necessary to realize the PEC water splitting. Obviously, this external bias produced by a power supply will lead to unnecessary consumption of electricity.

Recently, the unassisted PEC water splitting has become a research hotspot due to its more energy-saving and efficient characteristics for the STH conversion [34,35]. The namely "unassisted PEC water splitting" refers to the PEC process free from the external bias, in which all the energy used to split water molecules into hydrogen gas and oxygen gas is only provided by the incident sunlight.

Different from the conventional PEC water splitting with an external bias, the unassisted PEC water splitting is usually based on two novel strategies: to design tandem structures of photoanodes/photocathodes, and to construct hybrid devices of solar/PEC cells [36]. Specifically, for the former, a photoanode is coupled with a photocathode in a PEC cell to form a tandem structure (Fig. 1a). By this strategy, the photogenerated electrodes and holes can be produced simultaneously in both the photoanode and the photocathode under the incident light irradiation. Consequently, the photovoltages generated by the photoanode and the photocathode will be combined to provide sufficient voltage for water splitting. Therefore, this type of unassisted strategy can be described as the non-biased PEC water splitting. As for the latter strategy, a solar cell module is integrated with a PEC cell to construct a hybrid device. When this hybrid device is illuminated by solar light, the solar cell can provide the required bias for PEC cells to carry out the water-splitting reaction (Fig. 1b). On this basis, this second strategy can be regarded as the self-biased PEC water splitting.

Briefly, these two strategies can open up new avenues for the development of high-performance unassisted PEC water splitting technologies. Up to now, a serial of research has been carried out to develop these two types of technologies, including development on novel photoelectrode materials and design on unique device configurations [37-40]. However, there are still several critical aspects remaining unsolved, such as low STH efficiency of $<10 \%$ (i.e., the standard requirement for practical applications), high cost, and poor stability. Hence, more efforts should be made to further improve the performance of these unassisted PEC systems.

Based on these considerations, we believe that it is necessary to discuss in detail the future development of these two state-of-the-art technologies for PEC hydrogen production, and further propose feasible solutions to achieve the efficient unassisted PEC water splitting. Herein, we focus on the technological trends of the tandem structures and the hybrid devices, respectively.

For the former, three methods could be particularly feasible, including all-metal oxide photoelectrodes, nanoarray design, and surface modification. For the latter, device coupling, monolithic configuration, and multi-integration should be paid more attention to, in order to enhance the STH efficiency and stability of the hybrid systems (Fig. 2). In brief, all aspects mentioned above may attract the focus of research and become potential hot spots. At last, we hope that this perspective is valuable to inspire rational designs and developments for efficient and stable unassisted PEC water splitting.

\section{Tandem structure design of photoanodes and photocathodes}

The pioneering study of the tandem structure for unassisted PEC water splitting can be tracked back to 1977 [41]. Although it has developed for more than 40 years, the tandem structures are far from satisfying the commercial application, due to its low STH efficiency, poor stability, and high cost. To solve these issues, the following three effective strategies are important, as illustrated in Fig. 2: 

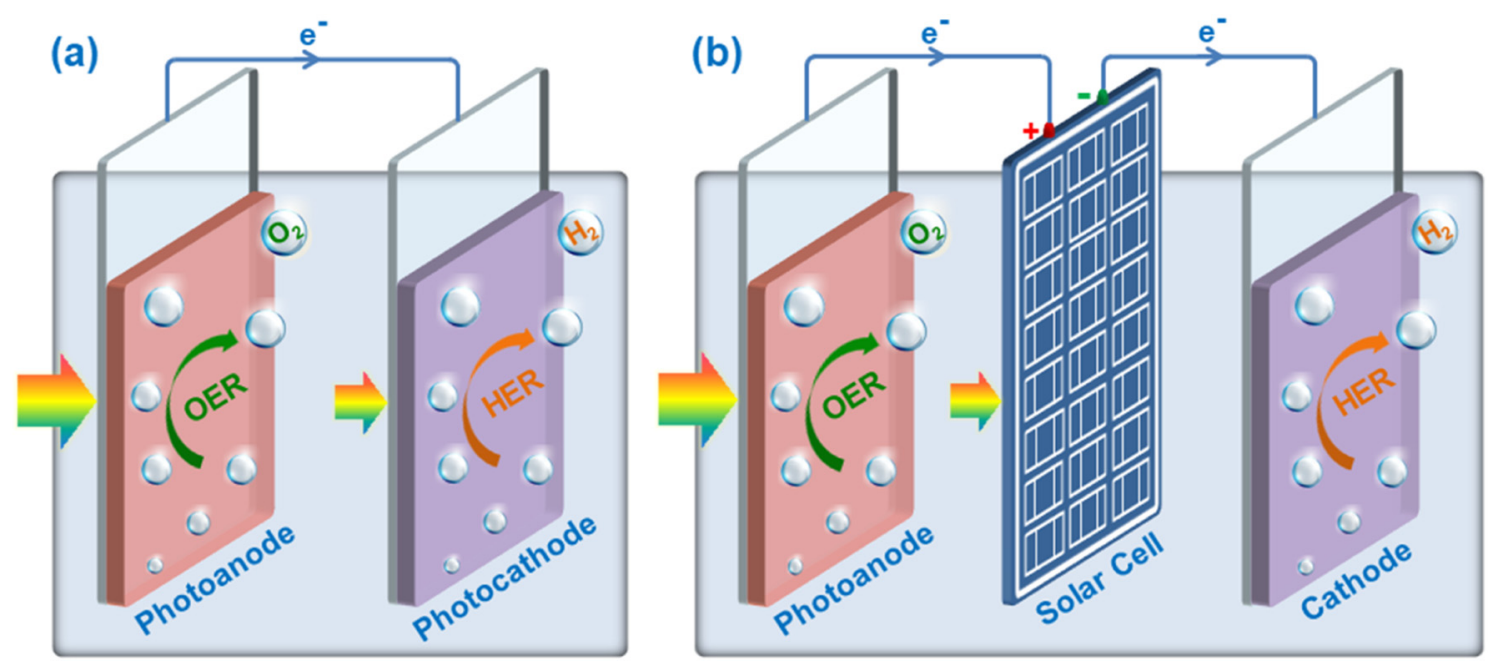

Fig. 1. Structural illustration of unassisted PEC water splitting cells based on (a) a tandem structure of photoanode/photocathode, and (b) a hybrid device of solar/PEC cell.

\subsection{All-metal oxide photoelectrodes}

Although some high efficiencies that are close to or even higher than $10 \%$ have been obtained in the laboratory, the photoelectrode materials utilized in these unassisted PEC tandem cells commonly involve $\mathrm{GaN}$, InN, InP, or Si-based semiconductors [42,43]. Obviously, some fatal shortcomings of these semiconductors, such as poor stability, high cost, and serious toxicity, make them unsuitable for largescale applications. Compared with the aforementioned materials, metal oxides are more promising for photoelectrode materials of tandem PEC cells in terms of good stability, low cost, non-toxicity, and facile fabrication.

Moreover, some other excellent features, such as $n / p$-type characteristics, wide distribution of the bandgap, suitable band edge positions for water electrolysis, and easily tunable electronic properties, show also better potential as photoelectrode materials. Firstly, metal oxides exhibit either intrinsic $n$-type (e.g., $\mathrm{TiO}_{2}, \mathrm{ZnO}$, and $\mathrm{WO}_{3}$ ) or $p$-type (e.g., $\mathrm{Cu}_{2} \mathrm{O}$ and $\mathrm{NiO}$ ) characteristics. Generally, the former can be used as the photoanodes, while the latter can be used as photocathodes. Thus, this provides a great possibility for simultaneously using both the $n$ type metal oxide as photoanode and the $p$-type metal oxide as a photocathode in a tandem PEC cell for unassisted solar-driven water splitting.
Secondly, metal oxides also own a wide-range distribution of bandgaps ( 3.4-2.0 eV), which can provide the appropriate band edge positions for water electrolysis and sufficient solar energy absorption. For instance, as a typical wide bandgap semiconductor, $\mathrm{TiO}_{2}$ has a bandgap of $\sim 3.2 \mathrm{eV}$, and its edges of the conduction band and valence band locate on the potentials of -0.1 and $+3.1 \mathrm{eV} v$ s. NHE, respectively. That is to say, $\mathrm{TiO}_{2}$ can provide photogenerated electrons and holes with sufficient energy to drive a water-splitting reaction under solar irradiation. On the other hand, as a narrow bandgap metal oxide, $\alpha-\mathrm{Fe}_{2} \mathrm{O}_{3}$ has a relatively small bandgap of $\sim 2.2 \mathrm{eV}$, which enables a high visible light absorption. Further, if a wide bandgap metal oxide (i.e., $\mathrm{TiO}_{2}$ ) is sensitized by a narrow metal oxide (i.e., $\alpha-\mathrm{Fe}_{2} \mathrm{O}_{3}$ ), the all-metal oxide photoelectrode material (i.e., $\alpha-\mathrm{Fe}_{2} \mathrm{O}_{3} / \mathrm{TiO}_{2}$ ) can not only provide the appropriate band edge positions for PEC water-splitting reaction but also exhibit the satisfactory visible light response.

Lastly, the electronic properties of metal oxides can be easily adjusted as well, such as by elemental doping or defect engineering, providing sufficient room for further enhancing the photoelectrodes. Consequently, these versatile advantages of metal oxides offer a large number of opportunities to construct the tandem PEC cells based on all-metal oxide photoelectrodes for unassisted PEC water splitting with high practical value.

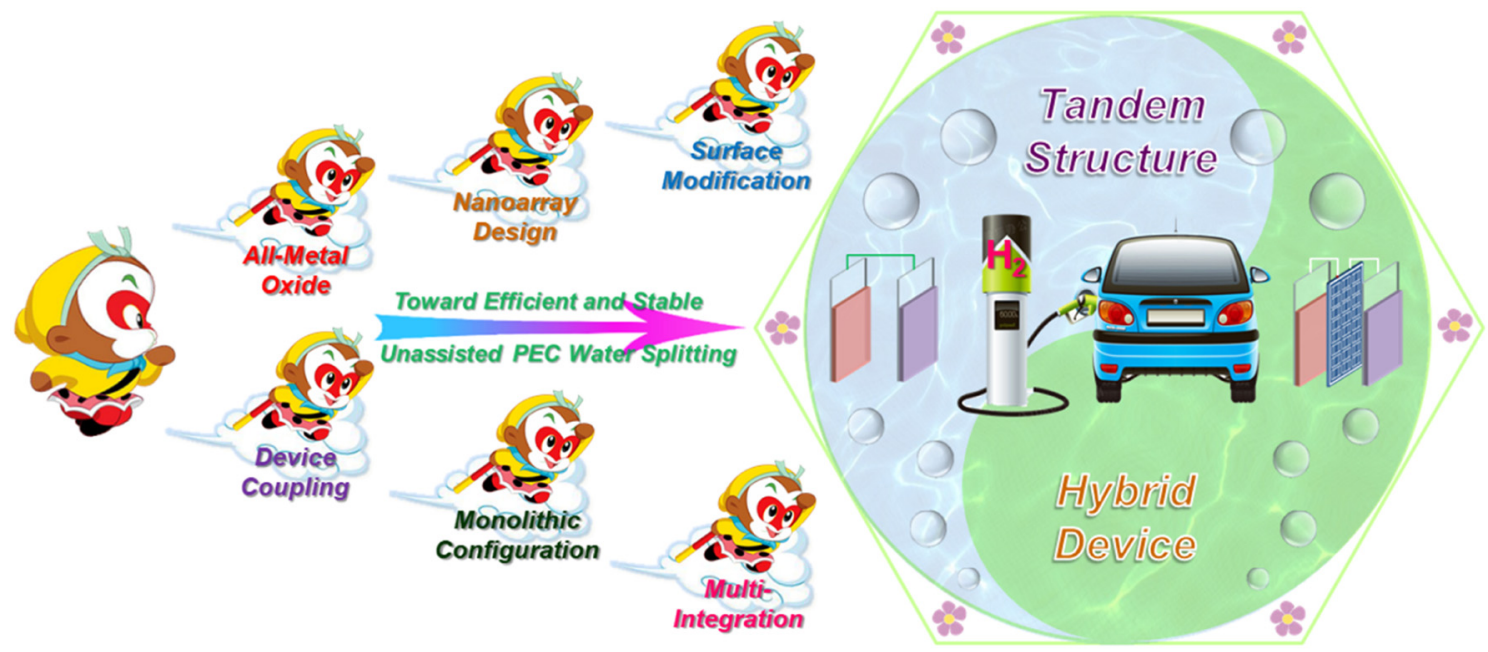

Fig. 2. Future technology trends of tandem structures and hybrid devices toward efficient and stable unassisted PEC water splitting. 


\subsection{Nanoarray design}

For efficient unassisted PEC water splitting, the successful suppression of the recombination of the photogenerated electrons and holes is one of the most critical factors. However, it is very challenging for the conventional mesoporous films, which are typically composed of nanoparticles (e.g., $\mathrm{TiO}_{2} \mathrm{P} 25$ powders), to meet this requirement. This is because the large number of grain boundaries in such materials would act as the charge-trapping sites, leading to a high recombination probability between photogenerated electrons and holes [44]. For this issue, using the highly ordered nanoarrays as photoelectrode materials can be a feasible solution to obtain the low exciton recombination, including nanotube arrays, nanorod arrays, nanowire arrays, and nanosheet arrays. In these nanostructured photoelectrodes, the highly ordered configurations can significantly shorten the diffusion distance of the photogenerated charge carriers to the surface of the material, resulting in the much-reduced recombination of photogenerated electrons/holes.

Except for the above-mentioned effect, the nanoarray designs also exhibit other unique merits. On the one hand, the highly anisotropic arrays can greatly increase the scattering of incident light in the photoelectrodes, thus enhancing the solar light harvesting. On the other hand, the nanoarrays can provide a large specific surface area, giving them more catalytically active sites and better contact with the electrolyte. Besides, the nanoarrays can also accelerate the release of gas bubbles generated at the surface of photoelectrodes, which is helpful to boost the dynamic process of PEC reactions. As a result, the nanoarray photoelectrodes are very promising potential candidates for highperformance unassisted PEC water splitting.

\subsection{Surface modification}

Apart from the above two points, the surface modification of photoelectrodes is also essential for achieving a more efficient and stable unassisted PEC water splitting based on the tandem structures. For instance, the OER commonly exhibits sluggish kinetics and a large overpotential, thus significantly limiting the overall STH efficiency of the whole unassisted system [45]. To overcome this problem, depositing cocatalysts onto the photoanode surfaces has been regarded as an effective method [36]. Especially, two emerging types of cocatalysts could exhibit the super-high catalytic activity, including metal oxyhydroxide (e.g., $\mathrm{NiOOH}, \mathrm{CoOOH}$, and $\mathrm{FeOOH}$ ), and single-atom catalysts (SACs) $[23,46]$.

For those photoelectrode materials that are easily photo-corroded, such as Si and CdS, the deposition of a highly stable protective layer can further enhance the durability of the tandem PEC systems. For example, graphitic carbon nitride ( $\mathrm{g}-\mathrm{CN}$ ) owns excellent physicochemical stability and can act as such protective layers to enhance the stability of $\mathrm{Si}$ or CdS photoelectrodes when it is coated onto them [47,48]. Furthermore, the in-situ deposition of g-CN can also keep the original micromorphologies of photoelectrodes, which is very beneficial to enhance the PEC performances as well [49].

\section{Hybrid devices of PEC/solar cells}

Except for the tandem structure design, integrating PEC cells with solar cells to form hybrid devices is another feasible way to achieve unassisted solar-driven water splitting. In such devices, the solar cell provides the bias for the PEC cells to carry out the water photolysis for hydrogen production under sunlight illumination.

Despite of the novel concept of hybrid devices, there are still some stumbling blocks on its road toward the commercialization, such as low STH efficiency, poor stability, and mismatching between PEC cells and solar cells, etc. In order to obtain the practical hybrid devices of $\mathrm{PEC} /$ solar cells, we believe that the following three constructive opinions should be paid full attention to (Fig. 2):

\subsection{Device coupling of PEC/solar cells}

In the early research stage, dye-sensitized solar cells (DSSCs) are frequently integrated with PEC cells for unassisted PEC water splitting due to their similar electrochemical behaviors [50,51]. However, the low open-circuit voltage $\left(V_{\mathrm{OC}}, \sim 0.5 \mathrm{~V}\right.$ ) produced by DSSCs is not sufficient for PEC cells, leading to a potential mismatching between solar cells and PEC cells, which greatly influences the STH efficiency of the hybrid devices. The novel perovskite solar cells (PSCs) not only generate a relatively high $V_{\mathrm{OC}}(0.9-1.1 \mathrm{~V})$, but also exhibit an excellent photoelectric conversion efficiency (>20\%) [52-54]. Consequently, introducing PSCs into the PEC/solar cell hybrid devices is a promising approach for achieving high-performance unassisted PEC water splitting [34].

Apart from the voltage coupling, the light absorption balance between PEC cells and solar cells should also be carefully considered to more sufficiently harvest solar energy. Specifically, if a PEC cell is placed in front of a solar cell, the photoelectrode will absorb the incident light with a wavelength smaller than its bandgap. Thus, the longwavelength part of the incident light would transmit through the photoelectrode and be absorbed by the solar cell. In this case, the PEC cell and the solar cell should be designed to be capable of absorbing different portions of the visible light to obtain a broad solar spectrum response. To realize this, two possible types of PEC photoelectrodes could be adopted, either on transparent conducting substrates or on mesh-shaped substrates.

\subsection{Monolithic configuration}

Two main strategies can be used to connect the PEC cell and the solar cell. One is the wire connection (i.e., external connection), and the other is the monolithic configuration (i.e., inner connection) [35]. In the first mode, the PEC cell and the solar cell are simply connected via external wires. Unfortunately, this technology leads to not only the loss of electric energy caused by the extra resistance but also the difficulty in light path design.

To obtain an efficient hybrid device, the monolithic configuration could be an ultimate solution. In this configuration, the PEC cell and the solar cell are integrated into a stand-alone device for unassisted PEC water splitting. This mode can decrease the device resistance, thus resulting in a high photocurrent and STH efficiency. But it needs to be considered that the solar cell in the monolithic hybrid device has to be immersed in and contact with the electrolyte, thus leading to a possible loss of stability for the whole system. Therefore, a reliable packaging technology, which can isolate the solar cell from the electrolyte and simultaneously provide the full contact between the PEC photoelectrode with the electrolyte, is essential to long-term stable work. This is particularly important for realizing stable unassisted PEC seawater splitting due to the serious corrosion capability of natural seawater.

\subsection{Multi-integration}

As mentioned, solar energy is greatly affected by day and night alternation, and change of weather and season, thus leading to intermittence and instability [19]. These disadvantages significantly limit the stable and continuous hydrogen production from unassisted PEC water splitting. Recently, a rising technology of converting and storing solar energy in the form of electrochemical energy could provide a solution to the aforesaid issue. Specifically, such devices, which are commonly composed of solar cells and electrochemical energy storage (EES) devices (e.g., lithium-ion batteries, sodium-ion batteries, lithium-sulfur batteries, supercapacitors, etc.), can convert solar energy into electricity, and subsequently store the electricity in EES units $[55,56]$. 


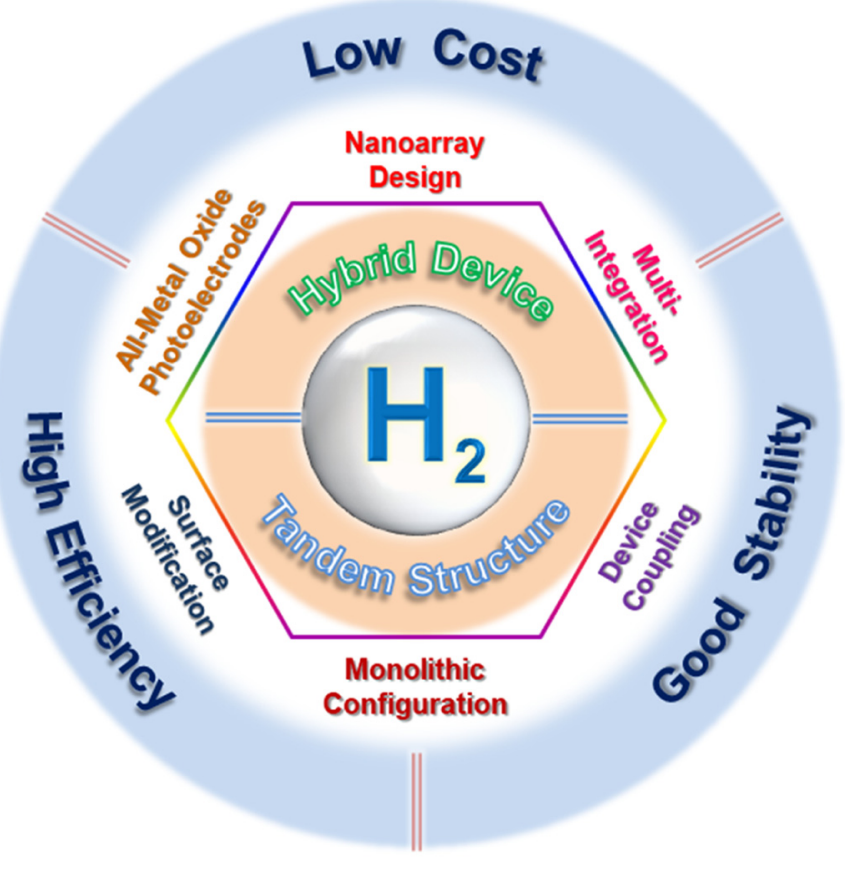

Fig. 3. Further developments on the tandem structure and the hybrid device toward more efficient, stable, and low-cost unassisted PEC water splitting.

For the multi-integration of PEC cells/solar cells/EES units, the solar cells can simultaneously provide the bias for PEC cells and charge for the EES units when the whole system is irradiated by sunlight. On the other hand, the EES units can discharge to drive the water-splitting reaction in PEC cells under the dark condition. Consequently, the multiintegration strategy can achieve an all-weather and 24-h water splitting for hydrogen generation, which is necessary for the large-scale industrial production. Besides, the multi-type energy harvesting, such as introducing nanogenerators into hybrid devices of PEC/solar cells, is also a feasible route to develop advanced unassisted PEC water splitting.

\section{Conclusion and outlook}

As a sustainable energy strategy, solar-driven water splitting for hydrogen evolution can simultaneously address the energy crisis and the environmental pollution [57-61]. Especially, unassisted PEC water splitting exhibits the greater development potential in comparison with other STH technologies. Specifically, unassisted PEC water splitting can be divided into two categories: the tandem structure of photoanode and photocathode and the hybrid device of solar/PEC cell. Although many efforts have been made, the unsolved problems still limit further development toward a more efficient, stable, and low-cost unassisted PEC water splitting (Fig. 3).

In this perspective article, we have predicted the future development trend in this field and proposed six feasible solutions to overcome current difficulties. On the one hand, all-metal oxide photoelectrodes, nanoarray design, and surface modification can provide more opportunities for tandem structure design to achieve a high-performance unassisted PEC water splitting. On the other hand, device coupling of PEC/ solar cells, monolithic configuration, and multi-integration are regarded as three promising ways for constructing PEC hybrid devices with high STH efficiency. In addition, attention should also be paid to the further cost reduction in unassisted PEC water splitting because this is a key step to the commercial applications.

At last, although there are still many challenges, we believe that unassisted PEC water splitting is very much anticipated in the near future, and will provide a sustainable technology for solar energy utilization.

\section{Declaration of Competing Interest}

The authors declare that there is no conflict of interests.

\section{Acknowledgements}

This work was supported by National Natural Science Foundation of China (No. 21905202), Innovative Research in the University of Tianjin (TD13-5077), and Developed and Applied Funding of Tianjin Normal University (135202XK1702), Australian Research Council (ARC) through Discovery Project (DP200100365), and Discovery Early Career Researcher Awards (DECRA, DE170100871).

\section{References}

[1] Y. Zheng, J. Liu, J. Liang, M. Jaroniec, S.Z. Qiao, Graphitic carbon nitride materials: controllable synthesis and applications in fuel cells and photocatalysis, Energy Environ. Sci. 5 (5) (2012) 6717-6731.

[2] H. Tian, J. Liang, J. Liu, Nanoengineering carbon spheres as nanoreactors for sustainable energy applications, Adv. Mater. 31 (50) (2019), 1903886.

[3] S. Devasahayam, Catalytic actions of $\mathrm{MgCO}_{3} / \mathrm{MgO}$ system for efficient carbon reforming processes, Sustain. Mater. Technol. 22 (2019), e00122.

[4] H.J. Chen, Y.L. Yang, M. Hong, J.G. Chen, G.Q. Suo, X.J. Hou, L. Feng, Z.G. Chen, Separable and recyclable meso-carbon@ $\mathrm{TiO}_{2} /$ carbon fiber composites for visible-light photocatalysis and photoelectrocatalysis, Sustain. Mater. Technol. 21 (2019), e00105.

[5] P.F. Wang, Y.F. Gu, L. Miao, J.H. Zhou, H. Su, A.Y. Wei, X.J. Mu, Y.Z. Tian, J.Q. Shi, H.F. $\mathrm{Cai}, \mathrm{CO}_{3} \mathrm{O}_{4}$ nanoforest/Ni foam as the interface heating sheet for the efficient solardriven water evaporation under one sun, Sustain. Mater. Technol. 20 (2019), e00106.

[6] H. Tian, J.H. Zhao, X.Y. Wang, L.Z. Wang, H. Liu, G.X. Wang, J. Huang, J. Liu, G.Q. Lu, Construction of hollow mesoporous silica nanoreactors for enhanced photooxidations over Au-Pt catalysts, Natl. Sci. Rev. (2020) https://doi.org/10.1093/nsr/ nwaa080.

[7] J.N. Li, Q.C. Zhang, J.M. Liu, M.R. Yu, H.Y. Ma, J.C. Yang, S. Ye, T.R. Reina, J. Liu, In-situ formation of carboxylate species on $\mathrm{TiO}_{2}$ nanosheets for enhanced visible-light photocatalytic performance, J. Colloid Interf. Sci. 577 (2020) 512-522.

[8] L.Q. Wang, J.M. Feng, Y.Y. Tong, J. Liang, A reduced graphene oxide interface layer for improved power conversion efficiency of aqueous quantum dots sensitized solar cells, Int. J. Hydrog. Energy 44 (1) (2019) 128-135.

[9] B.K. Sahu, Wind energy developments and policies in China: a short review, Renew. Sust. Energ. Rev. 81 (2018) 1393-1405.

[10] F.I. Petrescu, A. Apicella, R.V. Petrescu, S. Kozaitis, R. Bucinell, R. Aversa, T. AbuLebdeh, Environmental protection through nuclear energy, Am. J. Appl. Sci. 13 (9) (2016) 941-946.

[11] J.C. Hou, M.C. Cao, P.K. Liu, Development and utilization of geothermal energy in China: current practices and future strategies, Renew. Energy 125 (2018) 401-412.

[12] Z.L. Wang, T. Jiang, L. Xu, Toward the blue energy dream by triboelectric nanogenerator networks, Nano Energy 39 (2017) 9-23.

[13] L.X. Zhang, S.J. Tang, Y. Hao, M.Y. Pang, Integrated emergy and economic evaluation of a case tidal power plant in China, J. Clean. Prod. 182 (2018) 38-45.

[14] C. Rojas, M. Cea, A. Iriarte, G. Valdes, R. Navia, J.P. Cardenas-R, Thermal insulation materials based on agricultural residual wheat straw and corn husk biomass, for application in sustainable buildings, Sustain. Mater. Technol. 20 (2019), e00102.

[15] X.S. Li, C.G. Xu, Y. Zhang, X.K. Ruan, G. Li, Y. Wang, Investigation into gas production from natural gas hydrate: a review, Appl. Energy 172 (2016) 286-322.

[16] H. Su, J.H. Zhou, L. Miao, J.Q. Shi, Y.F. Gu, P.F. Wang, Y.Z. Tian, X.J. Mu, A.Y. Wei, L.H. Huang, S.Y. Chen, Z.J. Deng, A hybrid hydrogel with protonated $g-C_{3} N_{4}$ and graphene oxide as an efficient absorber for solar steam evaporation, Sustain. Mater. Technol. 20 (2019), e00095.

[17] J.H. Zhou, Y.F. Gu, Z.Y. Deng, L. Miao, H. Su, P.F. Wang, J.Q. Shi, The dispersion of Au nanorods decorated on graphene oxide nanosheets for solar steam generation, Sustain. Mater. Technol. 19 (2019), e00090.

[18] S.L. Zhang, N.D. Pham, T. Tesfamichael, J. Bell, H.X. Wang, Thermal effect on CZTS solar cells in different process of $\mathrm{ZnO} / \mathrm{ITO}$ window layer fabrication, Sustain. Mater. Technol. 18 (2018), e00078.

[19] B. Luo, D.L. Ye, L.Z. Wang, Recent progress on integrated energy conversion and storage systems, Adv. Sci. 4 (9) (2017), 1700104.

[20] J.T. Xu, Y.H. Chen, L.M. Dai, Efficiently photo-charging lithium-ion battery by perovskite solar cell, Nat. Commun. 6 (2015), 8103.

[21] L.Q. Wang, Y.Y. Tong, J.M. Feng, J.G. Hou, J. Li, X.G. Hou, J. Liang, G-C $\mathrm{C}_{3} \mathrm{~N}_{4}$-based films: a rising star for photoelectrochemical water splitting, Sustain. Mater. Technol. 19 (2019), e00089.

[22] L.Q. Wang, J. Han, J.M. Feng, X.W. Wang, D. Su, X.G. Hou, J.G. Hou, J. Liang, S.X. Dou, Simultaneously efficient light absorption and charge transport of $\mathrm{CdS} / \mathrm{TiO}_{2}$ nanotube array toward improved photoelectrochemical performance, Int. J. Hydrog. Energy 44 (59) (2019) 128-135.

[23] L.Q. Wang, W.P. Si, Y.Y. Tong, F. Hou, D. Pergolesi, J.G. Hou, L. Thomas, S.X. Dou, J. Liang, Graphitic carbon nitride ( $\mathrm{g}-\mathrm{C}_{3} \mathrm{~N}_{4}$ )-based nano-sized heteroarrays: promising materials for photoelectrochemical water splitting, Carbon Energy 2 (2) (2020) $223-250$. 
[24] L.Z. Ouyang, K. Chen, J. Jiang, X.S. Yang, M. Zhu, Hydrogen storage in light-metal based systems: a review, J. Alloys Compd. 829 (2020), 154597.

[25] J.O. Abe, A.P.I. Popoola, E. Ajenifuja, O.M. Popool, Hydrogen energy, economy and storage: review and recommendation, Int. J. Hydrog. Energy 44 (29) (2019) 15072-15086.

[26] K. Kay, Y. Hames, Two new control strategies: for hydrogen fuel saving and extend the life cycle in the hydrogen fuel cell vehicles, Int. J. Hydrog. Energy 44 (34) (2019) 18967-18980.

[27] Z.F. Liu, Q.G. Song, M. Zhou, Z.G. Guo, J.H. Kang, H.Y. Yan, Synergistic enhancement of charge management and surface reaction kinetics by spatially separated cocatalysts and p-n heterojunctions in Pt/CuWO $4 / \mathrm{CO}_{3} \mathrm{O}_{4}$ photoanode, Chem. Eng. J. 374 (2019) 554-563.

[28] Y.T. Li, Z.F. Liu, J. Zhang, Z.G. Guo, Y. Xin, L. Zhao, 1D/OD $\mathrm{WO}_{3} / \mathrm{CdS}$ heterojunction photoanodes modified with dual co-catalysts for efficient photoelectrochemical water splitting, J. Alloys Compd. 790 (2019) 493-501.

[29] J.G. Hou, S.Y. Cao, Y.Q. Sun, Y.Z. Wu, F. Liang, Z.H. Lin, L.C. Sun, Atomically thin mesoporous $\mathrm{In}_{2} \mathrm{O}_{3-\mathrm{x}} \mathrm{In}_{2} \mathrm{~S}_{3}$ lateral heterostructures enabling robust broadband-light photoelectrochemical water splitting, Adv. Energy Mater. 8 (9) (2018) 1701114.

[30] S.Y. Cao, Y.Z. Wu, J.G. Hou, B. Zhang, Z.W. Li, X.W. Nie, L.C. Sun, 3D porous pyramid heterostructure array realizing efficient photo-electrochemical performance, Adv. Energy Mater. 10 (5) (2020) 1902935.

[31] H.H. Cao, W.P. Si, W.L. Guo, T. Zhang, Y.H. Han, F. Hou, J. Liang, KCl flux suppresses surface recombinations of hematite photoanode for water oxidation, Surf. Innov. 8 (3) (2020) 130-137.

[32] W.P. Si, Z.P. Tehrani, F. Haydous, N. Marzari, I.E. Castelli, D. Pergolesi, T. Lippert, Yttrium tantalum oxynitride multiphases as photoanodes for water oxidation, J. Phys. Chem. C 123 (43) (2019) 26211-26217.

[33] S.R. Chen, C.L. Li, Z.Y. Hou, A novel in situ synthesis of $\mathrm{TiO}_{2} / \mathrm{CdS}$ heterojunction for improving photoelectrochemical water, Int. J. Hydrog. Energy 44 (47) (2019) 25473-25485.

[34] S.N. Yun, Y. Qin, A.R. Uhl, N. Vlachopoulos, M. Yin, D.D. Li, X.G. Han, A. Hagfeldt, Newgeneration integrated devices based on dye-sensitized and perovskite solar cells, Energy Environ. Sci. 11 (3) (2018) 476-526.

[35] Q. Chen, G.Z. Fan, H.W. Fu, Z.S. Li, Z.G. Zou, Tandem photoelectrochemical cells for solar water splitting, Adv. Phys. X 3 (1) (2018), 1487267.

[36] Y. Yang, S.W. Niu, D.D. Han, T.Y. Liu, G.M. Wang, Y. Li, Progress in developing metal oxide nanomaterials for photoelectrochemical water splitting, Adv. Energy Mater. 7 (19) (2017), 1700555.

[37] Z.M. Bai, J. Liu, Y.H. Zhang, Z. Huang, Y.K. Gao, X.T. Li, Y. Du, Unassisted solar water splitting using a $\mathrm{Cu}_{2} \mathrm{O} / \mathrm{Ni}(\mathrm{OH})_{2}-\mathrm{ZnO} / \mathrm{Au}$ tandem photoelectrochemical cell, J. Solid State Electrochem. 24 (2) (2020) 321-328.

[38] X. Shi, H. Jeong, S.J. Oh, M. Ma, K. Zhang, J. Kwon, I.T. Choi, I.Y. Choi, H.K. Kim, J.K. Kim, J.H. Park, Unassisted photoelectrochemical water splitting exceeding 7\% solar-to-hydrogen conversion efficiency using photon recycling, Nat. Commun. 7 (2016), 11943.

[39] X.J. Shi, K. Zhang, K. Shin, M. Ma, J. Kwon, I.T. Choi, J.K. Kim, H.K. Kim, D.H. Wang, J.H. Park, Unassisted photoelectrochemical water splitting beyond 5.7\% solar-tohydrogen conversion efficiency by a wireless monolithic photoanode/dyesensitised solar cell tandem device, Nano Energy 13 (2015) 182-191.

[40] V. Andrei, R.L.Z. Hoye, M. Crespo-Quesada, M. Bajada, S. Ahmad, M. De Volder, R. Friend, E. Reisner, Scalable triple cation mixed halide perovskite- $\mathrm{BiVO}_{4}$ tandems for bias-free water splitting, Adv. Energy Mater. 8 (25) (2018), 1801403.

[41] A.J. Nozik, Photochemical diodes, Appl. Phys. Lett. 30 (11) (1977) 567-569.

[42] K. Zhang, M. Ma, P. Li, D.H. Wang, J.H. Park, Water splitting progress in tandem devices: moving photolysis beyond electrolysis, Adv. Energy Mater. 6 (15) (2016), 1600602.

[43] Z.B. Luo, T. Wang, J.L. Gong, Single-crystal silicon-based electrodes for unbiased solar water splitting: current status, Chem. Soc. Rev. 48 (7) (2019) 2158-2181.
[44] P. Peerakiatkhajohn, J. Yun, S.C. Wang, L.Z. Wang, Review of recent progress in unassisted photoelectrochemical water splitting: from material modification to configuration design, J. Photon. Energy 7 (1) (2017), 012006.

[45] J.J. Huo, Y.L. Chen, Y. Liu, J.J. Guo, L. Lu, W.X. Li, Y. Wang, H. Liu, Bifunctional iron nickel phosphide nanocatalysts supported on porous carbon for highly efficient overall water splitting, Sustain. Mater. Technol. 22 (2019), e00117.

[46] T. He, S.M. Chen, B. Ni, Y. Gong, Z. Wu, L. Song, L. Gu, W.P. Hu, X. Wang, Zirconiumporphyrin-based metal-organic framework hollow nanotubes for immobilization of noble-metal single atoms, Angew. Chem. Int. Ed. 57 (13) (2018) 3493-3498.

[47] B.B. Wang, H.T. Yu, X. Quan, S. Chen, Ultra-thin $\mathrm{g}-\mathrm{C}_{3} \mathrm{~N}_{4}$ nanosheets wrapped silicon nanowire array for improved chemical stability and enhanced photoresponse, Mater. Res. Bull. 59 (2014) 179-184.

[48] R.N. Wang, J. Yan, M. Zu, S.Y. Yang, X. Cai, Q.Z. Gao, Y.P. Fang, S.S. Zhang, S.Q. Zhang, Facile synthesis of interlocking $\mathrm{g}-\mathrm{C}_{3} \mathrm{~N}_{4} / \mathrm{CdS}$ photoanode for stable photoelectrochemical hydrogen production, Electrochim. Acta 279 (2018) 74-83.

[49] J. Bian, Q. Li, C. Huang, J. Li, Y. Guo, M. Zaw, R.Q. Zhang, Thermal vapor condensation of uniform graphitic carbon nitride films with remarkable photocurrent density for photoelectrochemical applications, Nano Energy 15 (2015) 353-361.

[50] J.K. Kim, K. Shin, S.M. Cho, T.W. Lee, J.H. Park, Synthesis of transparent mesoporous tungsten trioxide films with enhanced photoelectrochemical response: application to unassisted solar water splitting, Energy Environ. Sci. 4 (4) (2011) 1465-1470.

[51] J. Brillet, J.H. Yum, M. Cornuz, T. Hisatomi, R. Solarska, J. Augustynski, M. Graetzel, K. Sivula, Highly efficient water splitting by a dual-absorber tandem cell, Nat. Photon. 6 (2012) 824-828.

[52] Y.C. Oiu, W. Liu, W. Chen, W. Chen, G.M. Zhou, P.C. Hsu, R.F. Zhang, Z. Liang, S.S. Fan, Y.G. Zhang, Y. Cui, Efficient solar-driven water splitting by nanocone $\mathrm{BiVO}_{4}$ perovskite tandem cells, Sci. Adv. 2 (6) (2016), e1501764.

[53] J. Su, H.K. Cai, X.F. Ye, X.J. Zhou, J.T. Yang, D. Wang, J. Ni, J. Li, J.J. Zhang, Efficient perovskite solar cells prepared by hot air blowing to ultrasonic spraying in ambient air, ACS Appl. Mater. Interfaces 11 (11) (2019) 10689-10696.

[54] X.J. Liang, Y. Cao, H.K. Cai, J. Su, J. Ni, J. Li, J.J. Zhang, Simulation and architectural design for Schottky structure perovskite solar cells, Acta Phys. Sin. 69 (5) (2020), 057901.

[55] Z.N. Tian, X.L. Tong, G. Sheng Y.L Shao, L.H. Yu, V. Tung J.Y. Sun, R.B. Kaner, Z.F. Liu, Printable magnesium ion quasi-solid-state asymmetric supercapacitors for flexible solar-charging integrated units, Nat. Commun. 10 (2019) 4913.

[56] Q. Zeng, Y.Q. Lai, L.X. Jiang, F.Y. Liu, X.J. Hao, L.H. Wang, M.A. Green, Integrated photorechargeable energy storage system: next-generation power source driving the future, Adv. Energy Mater. 10 (14) (2020), 1903930.

[57] J.G. Hou, Y.Z. Wu, S.Y. Cao, F. Liang, Z.S. Lin, Z.M. Gao, L.C. Sun, In situ phase-induced spatial charge separation in core-shell oxynitride nanocube heterojunctions realizing robust solar water splitting, Adv. Energy Mater. 7 (17) (2017), 1700171.

[58] J.G. Hou, Z. Wang, C. Yang, H.J. Cheng, S.Q. Jiao, H.M. Zhu, Cobalt-bilayer catalyst decorated $\mathrm{Ta}_{3} \mathrm{~N}_{5}$ nanorod arrays as integrated electrodes for photoelectrochemical water oxidation, Energy Environ. Sci. 6 (11) (2013) 3322-3330.

[59] J.G. Hou, C. Yang, H.J. Cheng, S.Q. Jiao, O. Takeda, H.M. Zhu, High-performance p$\mathrm{Cu}_{2} \mathrm{O} / \mathrm{n}$-TaON heterojunction nanorod photoanodes passivated with an ultrathin carbon sheath for photoelectrochemical water splitting, Energy Environ. Sci. 7 (11) (2014) 3758-3768.

[60] J.G. Hou, H.J. Cheng, O. Takeda, H.M. Zhu, Unique 3D heterojunction photoanode design to harness charge transfer for efficient and stable photoelectrochemical water splitting, Energy Environ. Sci. 8 (4) (2015) 1348-1357.

[61] Z.W. Li, J.G. Hou, B. Zhang, S.Y. Cao, Y.Z. Wu, Z.M. Gao, X.W. Nie, L.C. Sun, Twodimensional Janus heterostructures for superior Z-scheme photocatalytic water splitting, Nano Energy 59 (2019) 537-544. 